\title{
Analysis on Communication Influence of Official Microblogs
}

\author{
Nan Chen, ${ }^{1}$ Dingguo Yu $\left(\mathbb{D},{ }^{2,3}\right.$ Yijie Zhou, ${ }^{3}$ and Wenjuan Li $\mathbb{D I}^{1}$ \\ ${ }^{1}$ Qianjiang College, Hangzhou Normal University, Hangzhou 310018, China \\ ${ }^{2}$ College of Media Engineering, Communication University of Zhejiang, Hangzhou 310018, China \\ ${ }^{3}$ Key Lab of Film and TV Media Technology of Zhejiang Province, Communication University of Zhejiang, \\ Hangzhou 310018, China \\ Correspondence should be addressed to Dingguo Yu; yudg@cuz.edu.cn
}

Received 7 December 2020; Revised 22 January 2021; Accepted 31 January 2021; Published 12 February 2021

Academic Editor: Jorge E. Macias-Diaz

Copyright (c) 2021 Nan Chen et al. This is an open access article distributed under the Creative Commons Attribution License, which permits unrestricted use, distribution, and reproduction in any medium, provided the original work is properly cited.

As an innovative way for government affairs disclosure, official microblogs have been widely used in information release, public opinion listening, and public services, which become a model of "Internet + government affairs." The communication influence of official accounts is mainly related to the account's attention, activity, and the posted articles. Based on AHP, we design a multihierarchy-rank model to filter the factors and determine the most important factors related to the influence of official microblogs. This study explores the communication influence of official microblog from the perspectives of breadth, depth, and intensity; then, a three-dimensional integration of government microblogs influence calculation model for radiation, activity, and interaction is built and a monitoring and analysis system for the communication influence of government microblog is developed; finally, about 2,800 official accounts and more than 1.5 million articles in Zhejiang Province are monitored and analyzed. According to microblog verification, our proposed calculation model not only quantifies the communication influence of official microblogs but also analyzes their dynamics and timeliness. Therefore, it helps government departments to promote the healthy and orderly development of government microblogs.

\section{Introduction}

The new medias have entered the "Wei" era, and social medias represented by Microblog, WeChat, etc. have developed rapidly, which have become the primary platforms for the release and sharing of major information. As an innovative way for government affairs openness, the government affairs Microblog and WeChat public accounts have become more and more important, becoming the "standard configuration" for government affairs construction in many industries and regions, and being used to release information, collect opinions, listen to public opinion, and serve the public [1-3]. From the 43rd "China Internet Development Statistics Report" issued by China Network and Information Exchange Center [4], it can be obtained that, as of March 2020, the number of online government service users in China have reached 694 million, accounting for $76.8 \%$ of the overall netizens, of which the number of government microblogs certified by Sina platform have reached more than 139 thousand.
In social media, users establish connections with other users through the following function to build the topology of the social network and conduct information interactions by publishing, forwarding, and commenting information. The government affairs microblog is a bridge and link between the government and the masses. After the public becomes a fan through the following function, they can not only learn about government affairs information but also participate in government affairs interaction and obtain government services. Therefore, government affairs microblogs can make government decision-making more fair, open, and transparent, enabling people to express their opinions and suggestions without time and space restrictions and protecting people's right to speak. It collects public sentiment in time, and it is also a test of the government's administrative ability. This form can not only collect public sentiment in time but also test the government's administrative ability.

As a model of "Internet + government affairs," government affairs microblog has promoted the openness of government affairs through the application of new social 
media methods, enabling the sunny government and service-oriented government affairs to be indexed and evaluated. At present, the quality of the government affairs microblog platform is uneven, and some of them are proficient; how to evaluate and measure the effectiveness of its construction is imperative. On the one hand, it is necessary to summarize and analyze the effectiveness of the government affairs microblog platform construction at all levels of the province and relevant departments to find problems; on the other hand, the "Internet + government affairs" construction model needs to be further explored, so as to promote the openness of government affairs through Internet platforms, promote the Sunshine Government, and build a service-oriented government.

Affected by the massiveness, immediacy, and global interconnectivity of Internet platform data, as well as the complexity of social media network structures and the particularity of communication methods, the traditional media communication utility metrics and evaluation methods are completely incapable of evaluating the communication influence of government affairs microblogging platforms. Therefore, the study designs a data collector to extract related data from official accounts, such as articles and comments. Then, we propose a data analysis system, which can apply naive Bayes algorithm to filter the spam articles and users' comments. Next, analytic hierarchy process (AHP) is used to mine the data and extract relevant variables according to the hierarchy, which is called multihierarchy-rank. For example, in the study, we propose three factors of social media, such as network topology, user interaction behavior, and information content. And in the second layer, we can exact subfactors, such as user' comments, approval, due to the three elements mentioned above. Moreover, by applying big data mining and analysis techniques, our study constructs an evaluation system and corresponding computing models of government affairs microblog communication influence from the perspectives of breadth, depth, dimension, and effect. Last but not least, a government affairs microblog communication influence monitoring and analysis system software is integrated and developed to conduct an empirical analysis of the data collection and communication effects of government affairs microblog platforms established by government agencies at various levels in Zhejiang Province.

\section{Analysis of Research Status at Home and Abroad}

The three basic elements of social media include network topology, user interaction behavior, and information content [5]. The topology structure reflects the intuitive characteristics of user influence from the perspective of network topology; the user interaction behaviors (likes, reposts, comments, etc.) can reflect the generation and evolution of user influence; the information content (points of interest of user, topic content, novelty, dissemination time, etc.) can further reflect the details of the generation and evolution of influence [6]. Therefore, the main research direction today is measuring and evaluating the influence of microblog users from these three aspects.
The method of measuring the influence according to topology is mainly based on the measure of node degree, which mainly includes in-degree, out-degree, and degree centrality. The degree centrality [7] is used to measure the average influence of the current node on its neighbors; the higher the centrality of the node degree, the more the objects that focus on the user, the greater the influence of the node; the centrality of the degree reflects the local characteristics of the node. Besides, local centrality and local clustering coefficient not only consider the information of the node itself but also include some information of neighbor nodes.

Chen et al. established a part local centrality that collects multilevel neighbor information to sort the influence of nodes in the network via examining the degree information of the nodes with the nearest and second nearest neighbors [8]. The global evaluation criteria of nodes mainly comprise betweenness centrality [9], closeness centrality [10], and eigenvector centrality [11].

The PageRank algorithm and its extension method obtain the user's influence ranking by taking the user's interaction in the social network as an effective link and performing statistical ranking accordingly. For example, the Topic Leader Rank algorithm proposed by Wu et al. [12] can construct user behavior network computing user impact by using content and social attributes of microblog users; the Influence Rank algorithm [13] which considers the novelty of blog text itself and the link between blog posts; microblog-Rank algorithm [14] considering the user comment relationship; a calculation model of Sina microblog user influence based on information interaction proposed by $\mathrm{Yu}$ et al. In addition to the following relationships, users in social media also have actions such as likes, comments, and reposts, and the strength of influence between users can be better judged by analyzing these behaviors of users. By adding user reading habits and reposting characteristics in the process of calculating user influence, Mao et al. [15] comprehensively evaluated the influence. Based on a combination of network topology and historical user behavior, Tan et al. calculated the user influence [16]. On the basis of fully researching the four basic behaviors of users' forwarding, replying, reading, and copying, the calculation method of transition probabilities inside the network is given and the random walk model Multi-Rank based on multirelational networks is proposed to calculate users' single-topic influence and multitopic influence by Ding et al. [17].

Most social media content is short and concise, and latent dirichlet allocation (LDA) model is widely used as a topic extraction method for social media content analysis. By using LDA model to obtain the distribution of users in different topics and Gibbs to find out the strength of users' influence in different topics, Tang et al. [18] have obtained the user influence. According to Twitter's attention structure and user interest similarity, the Twitter Rank algorithm was proposed by Weng et al. [14] to calculate the user's influence on each topic; combining the social network topology with the LDM model, the social-relational topic model (SRTM) was designed by Guo et al. [19] to calculate the user's influence. In addition to the content of the information, the innovative nature of the information content and the time at which the information is released all play a role in 
determining the user's influence. Song et al. [20] calculate the user's influence by incorporating the novelty of information content into the topology of social networks.

Unlike ordinary microblog users, government affairs microblogs, as bridges and bonds between the government and the masses, have special functions. Some targeted research on the influence of government affairs microblogs have been carried out by scholars at home and abroad. According to the user's forwarding behavior, Chen et al. [21] summarized the basic characteristics and determinants of government microblog communication by using cluster analysis and other methods. Aiming at user's forwarding behavior, Li et al. [22] built a relatively complete government microblog reposting scale prediction model by performing feature extraction from multiple aspects such as user characteristics, content characteristics, and time characteristics. The essential characteristics of the provincial government microblog were revealed by Sha et al. [23] from the three aspects of overall network characteristics, cohesive subgroups, and centrality. By introducing complex network structure and modular public opinion indicators, Feng et al. [24] analyzed the government information microblog information diffusion channels and influence. Luo et al. [25] obtained the relationship between the characteristics of fans and the influence of government microblog through cluster analysis; Wang et al. constructed the government affairs microblog influence evaluation system by applying rough set method [26].

\section{Computational Model for Government Influence on Microblogs}

The influence of social media users on communication refers to the ability by posting, reposting, commenting, and liking online behaviors and their remarks to lead public opinion in social networks that influences the spread of network topics and other users' thoughts, network behavior, and speech. The influence of government affairs microblogs is similar to that of social media opinion leaders, but it is also unique. In the study, according to the relevant connotation and model of the theory of communication influence, and the application of big data mining and analysis methods, a comprehensive evaluation index system for the influence of government affairs microblog communication is defined.

3.1. Design of Evaluation Index System. The evaluation index system for the influence of government microblog communication includes three first-level indicators, six secondlevel indicators, and fourteen three-level indicators that have been refined layer by layer, including radiation, activity, and interaction. The following is a one-by-one explanation and description of the indicators at all levels.

Radiation indicates the radiation range of the information published by the government's microblog account and is the criterion for judging the breadth of microblog's spread. The higher the calculated value of radiation, the larger the area spread by the content published by the account, which users can be more concerned with. The indicator is refined into the following:
(1) The number of fans: Fans can see messages posted by the government affairs microblog on their own pages. Moreover, as a "blue V" authenticated user of Sina microblog, the government affairs microblog can actively send push messages to fans in the form of private messages. Fans can also further expand the visibility of the Microblog they follow through actions such as recommendations and comments.

(2) The number of forwarded posts: Forwarding behavior is the determinant of microblog's influence expansion. When the user reads and then forwards, after this process is cycled, the number of people who read the microblog will also increase. Thus, the number of forwarded posts is directly proportional to the radiation.

In the forwarding behavior, the total number of forwarding only indicates the number of forwards. However, in the three-level indicator, the total number of forwards, the average number of forwards, and the maximum number of forwards are included in the index system, which can reflect the radiation more intuitively.

Activity indicates the activeness of government microblog in a certain period of time, including the frequency of updating microblog and the number of logins to microblog. The higher the calculated value of the activity, the more standardized the operation of the account and the ability to regularly publish blog posts, and the better the effect of disseminating information. The indicator is refined into the following:

(1) The number of posts: This indicator includes the number of microblogs published by government affairs microblogs and the number of original microblogs published by it. The number of blog posts largely reflects the activity of an account. Particularly the original microblog has a higher topic level, which can arouse the interest of users, thus leading to an increase in user comments and retweets.

(2) Active days refer to the number of times the government affairs microblog account is active, which is an important determinant of Microblog's attention. A third-level indicator is introduced under the second-level indicator, which comprises the total number of active days, the number of active days in a shorter period of time, and the number of active days in a longer period of time. According to long-term observation data, it is known that blog posts published within 7 days are the golden time period for likes and comments. After exceeding this range, the numbers of likes and comments have decreased significantly. Besides, there will be no more users to like and comment on the blog post for more than 30 days. Therefore, a longer period of time is selected as 30 days, and a shorter period of time is selected as 7 days, which can better reflect the active degree of government affairs microblog. 
Interaction indicates the degree of interaction between government microblog users and other users. The main purpose of government affairs microblog is not only to disseminate government information, but also to collect and understand people's opinions to serve the people. Therefore, the degree of interaction is an important dimension for calculating the influence of government affairs microblogging. This indicator is refined into the following:

(1) Number of comments: When users generate their own thoughts or opinions on a government microblog, they will comment on it. The comments on the government affairs microblog largely reflect the public's views on certain social events and policies. The comments on Microblog can be recommented at by other users, or "@” other users to discuss. Therefore, the number of comments can reflect the level of interaction between government affairs microblog and other users. Not only is the number of comments expressed by its total number, but also the average and maximum numbers are included based on the total number, so that the number of reviews can be measured more accurately and intuitively.

(2) The number of likes, which is similar to the number of comments: When users have the same opinion on a microblog or express support for the content of the microblog but do not want to express their views, they often like the microblog. The government affairs microblog publishes some microblogs about hot events and policies. If the blog is liked, this can indicate that the information is recognized and supported by the public, which can also play a role in understanding public opinion. The number of likes is also divided into total likes, average likes, and maximum likes.

(3) The credibility from comments: When users are interested the contents released by the official account, they will comment on them. But at the same time, there will inevitably be some irrelevant information, which we call spam information. Therefore, we add another dimension to the interaction influence. We use the ratio of the number of valid comments to the total number of all comments to reflect the user's trust degree to this account.

3.2. Index Weight. The communication influence evaluation index system of the government microblog combines multiple evaluation methods, and then the experts will score to determine the weight of the indicators at all levels. By consulting experts in related fields, they will score according to the/importance of different indicators and the difficulty of obtaining data. Then the expert scoring results are processed to determine the weights of three different levels of indicators. The indicator weights are shown in Table 1.

3.3. Calculation Method of Transmission Influence. The calculation of government microblog's communication influence is based on the above evaluation indicators, including its interactive data (such as the number of likes and comments) and user information (such as the number of fans and the total number of posts).

The evaluation index system divides the communication influence into three levels of indicators and the data collected directly can only describe the current microblog characteristics. A normalized operation is used on the collected data to truly reflect the characteristics of government microblogs. The normalization method is calculated by comparing the user's current day's index value with the highest value of the index and as

$$
I_{k k}=\frac{\mathrm{d}_{k}}{\mathrm{~d}_{\max }} .
$$

If the total comment $I 11$ is taken as an example, then

$$
I_{11}=\frac{\text { total number of comments on government affairs microblog }}{\text { the maximum total number of comments on all government microblogs collected }} .
$$

The calculation of communication influence needs to be added step by step according to the indicator weight table. The index value of the lowest level indicator can be calculated directly, and the secondary indicator (such as the number of likes) is calculated by weighting the subindicator to which it belongs. By analogy, the calculated value of the first-level indicator (such as the degree of interaction) is calculated by weighting its two second-level indicators and then added. Finally, the calculation of the communication influence is obtained by adding the weighted calculations of the three dimensions. The calculation formula for the second-level indicator is shown in Formula (3):

$$
I_{x}=I_{11} \times \lambda_{a}+I_{12} \times \lambda_{b}+I_{13} \times \lambda_{c} .
$$

The calculation formula for the first-level indicator is

$$
I=I_{1} \times \theta_{a}+I_{2} \times \theta_{b} .
$$

The formula for calculating the communication influence is

$$
\mathrm{IF}=0.4 \times R+0.3 \times A+0.3 \times I .
$$

\section{Monitoring and Analysis System for the Communication Influence of Official Microblogy}

4.1. Structural Design of System Functions. According to the designed calculation model of communication influence of the government microblog, we have developed a 
TABLE 1: Temperature and wildlife count in the three areas covered by the study.

\begin{tabular}{|c|c|c|c|c|c|}
\hline $\begin{array}{l}\text { First-level } \\
\text { index }\end{array}$ & Weights & Second-level index & Weights & Third-level index & Weights \\
\hline Radiation $R$ & $\beta_{1}=0.4$ & $\begin{array}{l}\text { Number of forwards } \\
\qquad R_{1} \\
\text { Number of fans } R_{2}\end{array}$ & $\begin{array}{l}\theta_{1}=0.6 \\
\theta_{2}=0.4\end{array}$ & $\begin{array}{c}\text { Total number of forwards } R_{11} \\
\text { Average number of forwards } R_{12} \\
\text { Maximum number of forwards } R_{13} \\
\text { None }\end{array}$ & $\begin{aligned} & \lambda_{1}=0.6 \\
& \lambda_{2}=0.3 \\
& \lambda_{3}=0.1 \\
& \text { None }\end{aligned}$ \\
\hline Activity $A$ & $\beta_{2}=0.3$ & Number of posts $A_{1}$ & $\begin{array}{l}\theta_{3}=0.7 \\
\theta_{4}=0.3\end{array}$ & $\begin{array}{c}\text { Total number of posts } A_{11} \\
\text { Number of originals } A_{12} \\
\text { Total activity } A_{21} \\
\text { Total activity in a long period } A_{22} \\
\text { Total activity in a short period } A_{23}\end{array}$ & $\begin{array}{l}\lambda_{4}=0.5 \\
\lambda_{5}=0.5 \\
\lambda_{6}=0.5 \\
\lambda_{7}=0.2 \\
\lambda_{8}=0.3\end{array}$ \\
\hline \multirow[t]{2}{*}{ Interaction $I$} & \multirow[t]{2}{*}{$\beta_{3}=0.3$} & $\begin{array}{l}\text { Number of comments } \\
\qquad I_{1}\end{array}$ & $\theta_{6}=0.5$ & $\begin{array}{c}\text { Total number of comments } I_{11} \\
\text { Average number of comments } I_{12} \\
\text { Maximum number of comments } I_{13} \\
\text { Total number of likes } I_{21} \\
\text { Average number of likes } I_{22} \\
\text { Maximum number of likes } I_{23} \\
\text { Ratio of the number of valid comments to total number of }\end{array}$ & $\begin{array}{l}\lambda_{9}=0.5 \\
\lambda_{10}=0.3 \\
\lambda_{11}=0.2 \\
\lambda_{12}=0.5 \\
\lambda_{13}=0.3 \\
\lambda_{14}=0.2\end{array}$ \\
\hline & & Credibility $I_{3}$ & $\theta_{7}=0.2$ & comments $I_{31}$ & $\lambda_{15}=1$ \\
\hline
\end{tabular}

communication influence monitoring and analysis system software of official microblogs, comprising three major modules of microblog data directional collection, analysis engine, and visual analysis, and the software structure is shown in Figure 1.

The microblog data directional collection is mainly used to collect the data of the microblog, which can automatically collect microblog data of monitored accounts according to the configuration, comprising the content of each blog post and the corresponding statistics of the blog posts, likes, reposts, and comments. In addition, it automatically updates the statistics of blog posts on a regular basis. The data directional collection module is mainly composed of two parts of the acquisition management module and the microblog crawler. The collection management module can centrally manage and control the numerous microblog crawlers deployed in a distributed manner; the microblog crawler can realize the automatic collection of microblog web page information and can intelligently parse the web page content, automatically deduplicate, denoise, and extract the specified information content, and finally convert it into structured data.

The analysis engine can automatically perform statistical analysis on the collected microblog data and calculate the monitored influence of the government microblog account based on the defined government influence microblog's propagation influence calculation model. The visual analysis module presents the calculation results with intuitive images such as graphs and tables.

4.2. Experiment and Result Analysis. First, more than 2,600 official microblog accounts in Zhejiang Province are collected, involving public security, culture, tourism, transportation, taxation, party and government agencies, social teams, and other industries. Then, the monitoring and analysis system for the communication influence of government microblogs is used to monitor and analyze them. The system automatically collected the likes, forwards, and comments of each article published by these accounts in the past 3 months. As of December 30, 2019, the system has collected a total of 1.03 million microblog articles and about 3.56 million comments. Based on this data, the influence of official microblog account monitored by the system is calculated according to the government influence microblog communication influence calculation model proposed in the study.

The information of Top 30 government affairs microblog accounts obtained by the model is shown in Table 2 .

From the data obtained from the analysis system, it can be seen that the government microblogs of government agencies, tourism, and public security have a stronger influence on social media users. The government department microblogs, which are officially set by the People's News Government Office, rank first in the influence of Zhejiang government microblogs. The articles published by the official microblogs of government agencies at all levels cover a wide range of fields, containing culture, scenery, education, security, and people's livelihood. Thus, most users will use this type of government microblogs to learn what has happened at the present moment. Therefore, the microblogs of the government agency sector rank first. Next, the influence of travel microblogs ranks second. Obviously, Zhejiang has always been famous for its rich tourism resources, beautiful scenery, and complete supporting facilities. Therefore, tourists will learn about tourism projects and activities of Zhejiang Province from the microblogs' articles of tourism departments. What is more, the microblogs' influence of public security ranks third. Users can obtain safety information and knowledge by such microblogs and try their best to prevent accidents.

In addition, the other influential factors of government affair microblogs' communication influence in Zhejiang Province are shown in Figure 2. It obviously can be seen that microblog's fan number, article number, and interaction 


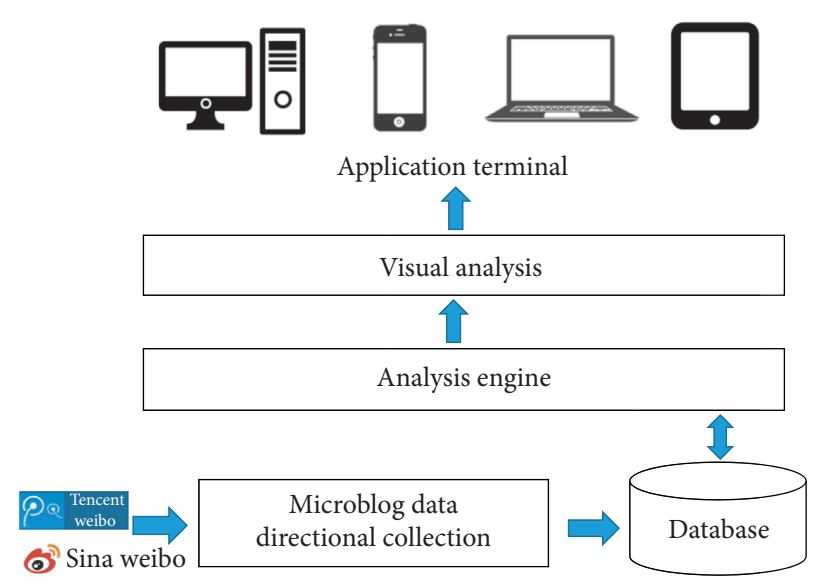

FIgURE 1: The structure of influence monitoring and analysis system.

TABLE 2: Communication influence ranking table.

\begin{tabular}{|c|c|c|c|c|c|}
\hline Influence ranking & Names of official microblogs & Radiation index & Activity index & Interaction index & Influence index \\
\hline 1 & Hangzhou publicity & 58707 & 54880 & 23095 & 48251 \\
\hline 2 & Yuhang publicity & 59621 & 38068 & 27480 & 48043 \\
\hline 3 & Zhejiang travel & 58784 & 31920 & 28460 & 47088 \\
\hline 4 & Zhejiang fire & 37734 & 30061 & 24168 & 33016 \\
\hline 5 & Hangzhou police & 33675 & 43601 & 26124 & 32728 \\
\hline 6 & Zhuji education & 38379 & 17242 & 22865 & 31521 \\
\hline 7 & Hangzhou travel & 32525 & 40410 & 23576 & 30944 \\
\hline 8 & Zhejiang committee & 25989 & 47662 & 29668 & 29586 \\
\hline 9 & Zhejiang publicity & 27341 & 42840 & 27392 & 29190 \\
\hline 10 & Peace wenzhou & 24362 & 26036 & 38835 & 28626 \\
\hline 11 & Ningbo police & 22638 & 67075 & 24911 & 28534 \\
\hline 12 & Ningbo publicity & 20189 & 59135 & 28554 & 27146 \\
\hline 13 & Jinhua police & 18318 & 37771 & 31199 & 24237 \\
\hline 14 & Chunan publicity & 19100 & 44447 & 24486 & 23611 \\
\hline 15 & Peace jianggan & 14902 & 21300 & 42578 & 23433 \\
\hline 16 & Jiaxing fire & 23238 & 22231 & 22755 & 22983 \\
\hline 17 & Bingjiang publicity & 18732 & 21173 & 31545 & 22620 \\
\hline 18 & Jianggan publicity & 19169 & 36183 & 23052 & 22272 \\
\hline 19 & Shangcheng publicity & 21625 & 30005 & 19799 & 22103 \\
\hline 20 & Zhejiang police & 17683 & 34887 & 25303 & 21858 \\
\hline 21 & Hanzhong urban management & 14950 & 39697 & 28278 & 21621 \\
\hline 22 & Wenzhou traffic & 14553 & 43372 & 26931 & 21481 \\
\hline 23 & Peace west lake & 19404 & 17440 & 26016 & 21029 \\
\hline 24 & Huangyan publicity & 16850 & 12931 & 31121 & 20395 \\
\hline 25 & Shangcheng petition & 7754 & 15755 & 29003 & 20186 \\
\hline 26 & Haining publicity & 14219 & 29224 & 28984 & 20142 \\
\hline 27 & Zhejiang law & 21272 & 9841 & 20380 & 19669 \\
\hline 28 & Yiwu fire & 7025 & 20310 & 26254 & 19616 \\
\hline 29 & Qiandao lake travel & 15845 & 33727 & 20344 & 19224 \\
\hline 30 & Zhejiang weather & 12893 & 38300 & 23701 & 18935 \\
\hline
\end{tabular}

number make great contribution to communication influence of microblogs. Those with a large number of fans, articles, and interactions (likes, comments, and forwards) commonly have great influence on social media users. To be fair, compared with the three factors, the article number of microblogs has a weaker impact on communication influence of microblogs.

All in all, these microblogs of strong communication influence have the characteristics of high quality contents, large numbers of fans, fast update speed, frequent 


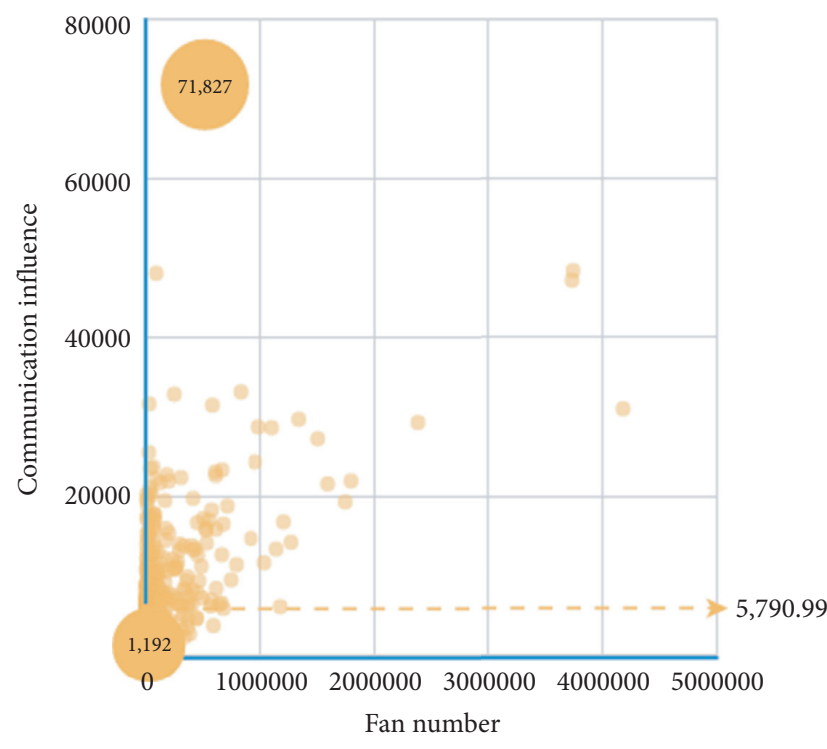

(a)

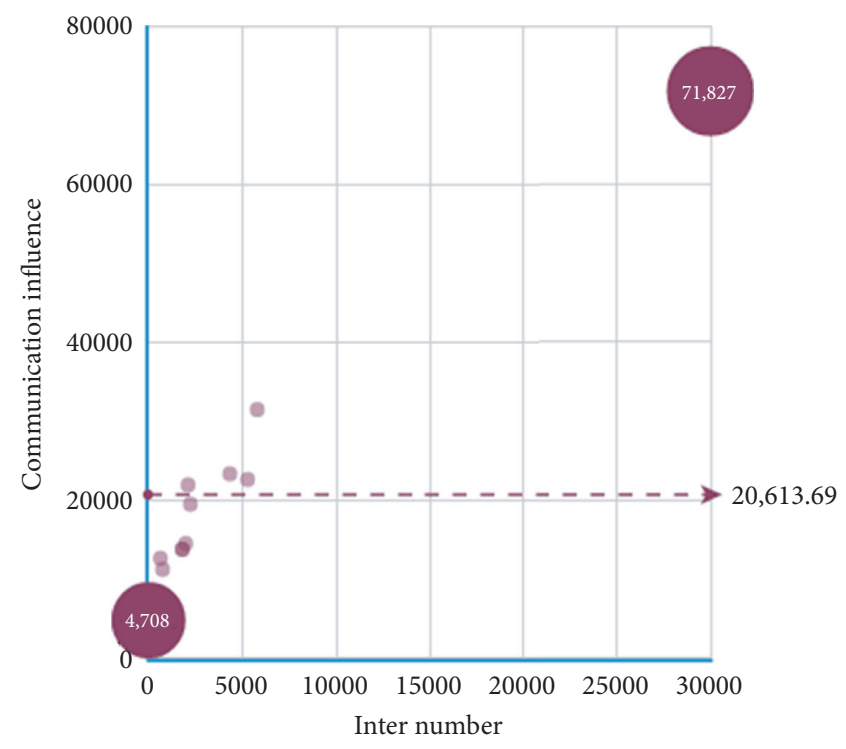

(b)

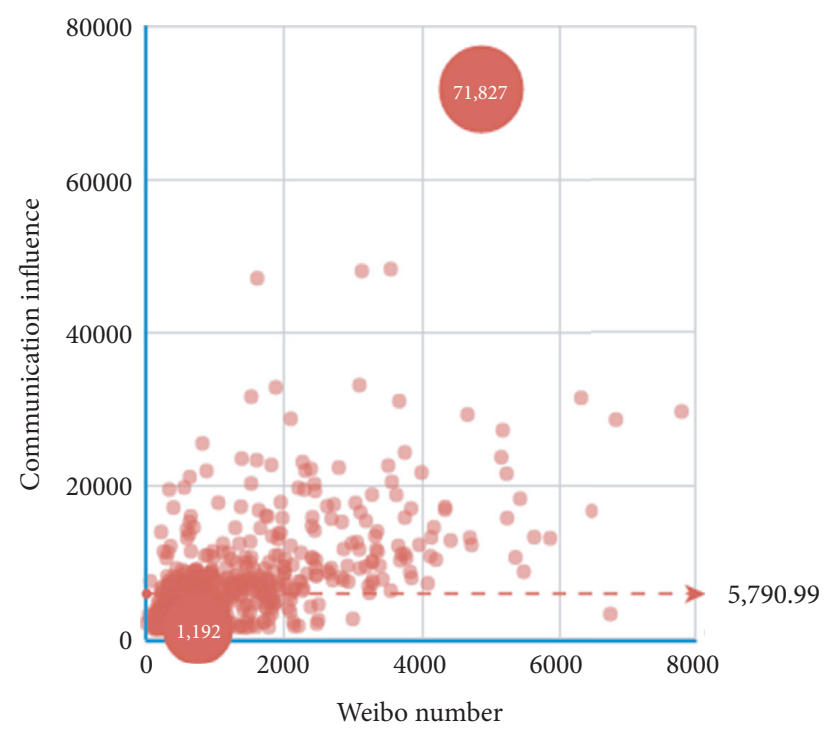

(c)

FIGURE 2: Influential factors of government affairs microblogs' communication influence.

interaction, etc., which attract the attention of the public, thus having a higher impact.

\section{Conclusions}

The government affairs microblog with a wide spread and large influence will have a profound impact on social issues, citizen deliberations, and popularization of policies. However, the spread and operation of various government microblogs are different, and homogeneous government microblogs still exist. In response to this situation, to better perform quantitative analysis and evaluate the effect and influence of government affairs microblogs, a set of government affairs microblog communication effect evaluation system and a monitoring and analysis system of micro government communication influence is designed in this study, thus analyzing and judging the government affairs microblog's communication effect and influence through a scientific and effective method. According to the practical data analysis results, the trinity model of governmental microblog communication impact based on radioactivity, activity, and interaction designed in this study can better analyze and evaluate the communication influence of government affairs microblog; besides, it can also reflect the dynamic and timeliness of communication influence of government affairs microblog. Moreover, the results indicate that the number of fans and posts is an important factor influencing the influence of microblog accounts, but it is not a decisive factor.

\section{Data Availability}

The data in this paper are derived from Sina Web account. 


\section{Conflicts of Interest}

The authors declare that they have no conflicts of interest.

\section{Acknowledgments}

This work was supported by the MOE (Ministry of Education in China) Project of Humanities and Social Sciences (no. 18YJA860020), the National Natural Science Foundation of China (no. 61702151), and the Key Research and Development Program of Zhejiang Province, China (no. 2019C03138).

\section{References}

[1] J. Chen and Y. Qin-Jian, "A review of administrative microblogging study in China and abroad," Information Science, vol. 32, no. 6, pp. 156-161, 2014, in Chinese.

[2] J. Yu, Z. Xie, and X. Ma, "Development context and future prospect: ten years of research on new media of Chinese government affairs," Academic Library and Information Science, vol. 38, no. 05, pp. 122-129, 2020, in Chinese.

[3] L. Zhao, "The development and communication of new media of government affairs," Reporter Observed, vol. 24, pp. 22-23, 2020, in Chinese.

[4] CNNIC, 45th China Internet Development Statistics Report, China Internet Network Information Central, Beijing, China, 2020, in Chinese.

[5] Z. M. Han, Y. Chen, W. Liu, B. H. Yuan, M. Q. Li, and D. G. Duan, "Research on node influence analysis in social networks," Ruan Jian Xue Bao/Journal of Software, vol. 28, no. 1, pp. 84-104, 2017, in Chinese.

[6] C. X. Wang, X. H. Guan, T. Qin, and W. Li, "Who are active? An in-depth measurement on user activity characteristics in sina microblogging," in Proceedings of the GLOBECOM. Piscataway, pp. 2083-2088, IEEE, Anaheim, CA, USA, December 2012.

[7] L. C. Freeman, "Centrality in social networks conceptual clarification," Social Networks, vol. 1, no. 3, pp. 215-239, 1979.

[8] D. Chen, L. Lü, M.-S. Shang, Y.-C. Zhang, and T. Zhou, "Identifying influential nodes in complex networks," Physica A: Statistical Mechanics and Its Applications, vol. 391, no. 4, pp. 1777-1787, 2012.

[9] L. C. Freeman, "A set of measures of centrality based on betweenness," Sociometry, vol. 40, no. 1, pp. 35-41, 1977.

[10] G. Sabidussi, "The centrality index of a graph," Psychometrika, vol. 31, no. 4, pp. 581-603, 1966.

[11] K. Stephenson and M. Zelen, "Rethinking centrality: methods and examples," Social Networks, vol. 11, no. 1, pp. 1-37, 1989.

[12] W. U. Xian-Hui, H. Zhang, X.-J. Zhao et al., "Mining algorithm of microblogging opinion leaders based on userbehavior network," Application Research of Computers, vol. 32, no. 4, 2015, in Chinese.

[13] S. Xiao-Dan, C. H. I. Yun, K. Hino et al., "Identifying opinion le aders in the blogosphere," in Proceedings of the Sixteenth ACM Conference on Information and Knowledge Management, 2007, pp. 971-974, ACM Press, New York, NY, USA, January 2007.

[14] L. Yan, L. Hua-Xian, L. Xue-Qiao et al., "Hot topic propagation model and opinion leader identifying model in microblog network," Abstract and Applied Analysis, vol. 36, no. 2, pp. 360-367, 2013.
[15] J. X. Mao, Y. Q. Liu, H. M. Zhang, and S. P. Ma, "Social influence analysis for micro-blog user based on user behavior," Chinese Journal of Computers, vol. 37, no. 4, pp. 791-800, 2014.

[16] Z. M. Han, Y. Chen, W. Liu, B. H. Yuan, M. Q. Li, and D. G. Duan, "Research on node influence analysis in social networks," Ruan Jian Xue Bao/Journal of Software, vol. 28, no. 1, pp. 84-104, 2017, in Chinese.

[17] Z. Ding, B. Zhou, Y. Jia et al., "Topic influence analysis based on the multi-relational network in microblogs," Journal of Computer Research and Development, vol. 50, no. 10, pp. 2155-2175, 2013.

[18] J. Tang, J. Sun, C. Wang, C. Wang, and Z. Yang, "Social influence analysis in large-scale networks," in Proceedings of the 15th ACM SIGKDD Int'l Conf. on Knowledge Discovery and Data Mining (KDD 2009), J. Elder and F. S. Fogelman, Eds., ACM Press, New York, NY, USA, pp. 807-816, June 2009.

[19] W. Guo, S. Wu, L. Wang, and T. Tan, "Social-Relational topic model for social networks," in Proceedings of the 24th ACM Int'l Conf. on Information and Knowledge Management (CIKM 2012), pp. 1731-1734, ACM Press, New York, NY, USA, October 2015.

[20] X. Song, Y. Chi, H. Koji et al., "Identification opinion leader in the blogosphere," in Proceedings of the 16th ACM Conf on Information and Knowledge Management, pp. 971-974, ACM, New York, NY, USA, January 2007.

[21] R. Chen and Y. Liu, "Study on model of government micro blogs information transmission based on forward behavior," E-goverment, vol. 7, pp. 108-117, 2017, in Chinese.

[22] Q. Li, J. Jiang, Li Ying, and Y. Liu, "The retweeting scale classification prediction of government microblogs in China," Journal of Intelligence, vol. 37, no. 1, pp. 95-99, 2018, in Chinese.

[23] S. Zhongyong and Y. Su, "Social network analysis of provincial government micro blogs in China," Jinan Journal (Philosophy and Social Sciences), vol. 6, pp. 125-132, 2018, in Chinese.

[24] H. Feng, C. Lei, C. Yang, and M. Yuan, "Research on the influence of government affairs microblog based on modularity of complex network," Journal of Changzhou University (Social Science Edition), vol. 17, no. 6, pp. 37-42, 2016, in Chinese.

[25] Y. Luo, G. Hu, and M. Lu, "Study on government affairs micro-blog influence and fans' characteristic relation," $E$ goverment, vol. 12, pp. 82-89, 2017, in Chinese.

[26] S. Wang, Y. Ge, and Q. Zhao, "Evalution method and empirical study of government micro-blog influence based on rough set theory," Mathesmatics in Practice and Theory, vol. 48 , no. 22, pp. 76-84, 2018, in Chinese. 